\title{
A Case Series Of Poorly Differentiated Carcinoma Endometrium IHC Confirmed Reported at AHRCC Cuttack, State Odisha, Country India
}

\author{
S.Spattnaik,J.J Mohapatra, J.Parija, L.Sarangi,R.Das, N.Rout,S.K Giri,S.Samantray, N.Panda,P.Devi, P.K Das \\ B.L Nayak, M.Rmohapatra,A.K Padhi,S.Panda \\ Dept - Gynaecology Oncology, Institute- Ahrcc, City Cuttack, State -Odisha, Country India
}

DOI: $10.29322 /$ IJSRP.10.03.2020.p9920

http://dx.doi.org/10.29322/IJSRP.10.03.2020.p9920

\begin{abstract}
Endometrial carcinoma has a high morbidity in advanced countries of eastern Europe and usa and japan, here its morbidity has increased in recent years . therefore it has become increasingly important to understand the oncogenetic mechanism and prognostic factors of endometrial cancer. It has reported that the gradeof differentiation is one of the critical prognostic factors. Various studies reveal 5 yrs survival decreases ith lower differentiation grades.92\% G1 well differentiated, and $86 \%$ and $74 \%$ respectively in G2 and G3.The poorly differentiated cancer have a higher rate of metastasis, recurrence and loer over all survival. Diagnosis of poorly differentiated cancer of endometrium may sometimes, be difficult and can be confused with other malignancies.here we report to cases of poorly differented carcinoma, confirmed by IHC
\end{abstract}

Index Terms- ET- ENDOMETRIAL THICKNESS, SOL- SPACE OCCUPYING LESION, BPLND- B/L PELVIC LYMPHADENECTOMY, BSO-B/LSALPINGOOPHERECTOMY, PDL-1 PROGRAMWDEATH LIGAND

\section{INTRODUCTION}

st case-

1 History - 72 yrs female presented to opd with ,c/o - postmenopausal bleeding for 2 mths, no watery discharge , no pain abdomen.M/h- attained menopause $20 \mathrm{yrs}$ back.O/H - p4l4/ all vd, lcb $32 \mathrm{yrs}$, Not sterilised .F/h - no family history of cancer .T/Hno history of any hormonal therapy

On/examination- average built, good nutritional status no scln, absensce of pedal oedema ,b/l- breasts normal on examination.P/A soft ned .P/S- cx and vagina healthy.P/VP/R- Uterus bulky rv, b/l fornices free

\section{INVESTIGATIONS -}

- PAP SMEAR- atypical squamous cell can”t exclude hsil

- USG - ET- 18mm, no sol 


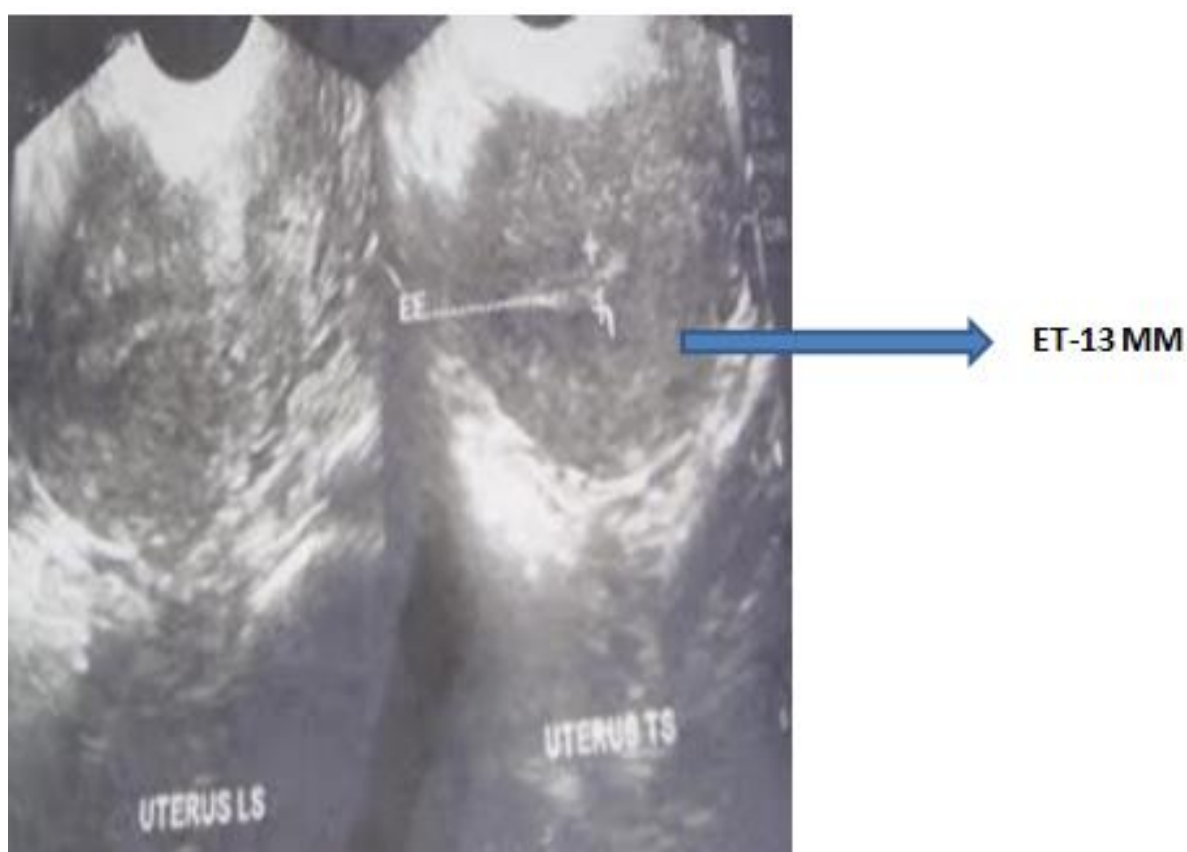

- DIAGNOSTIC HYSTEROSCOPY ENDOMETRIAL BIOPSY-growth $(2 \times 2)$ seen in posterior wall in the fundus

- EB- Endometrial stroma with fragmented glands with areas haemorrage Plan - hysterectomy

- $\quad$ Procedure - ndvh
IOP
- uterus bulky $+\mathrm{b} / \mathrm{l}$ tubes and ovaries
normal

- Cut section - 2x2 cm growth at fundus ,> 50\% myo-invasion, myometrium and could not be differentiated, endocervical cavity normal

\section{HPS}

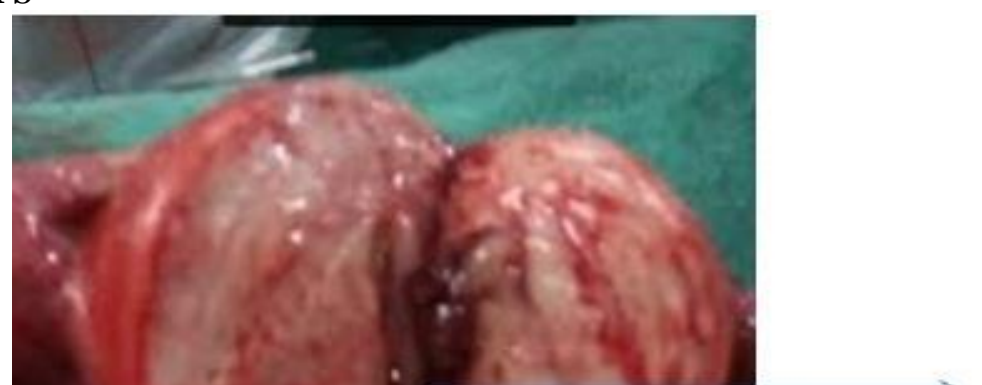

$\mathrm{C} / \mathrm{S}-$ Growth of $2 \times 2 \mathrm{~cm}$ tan - white

- cervix- chronic cervicitis and free of tumor

- $b / 1$ tubes and ovaries were free of tumor 

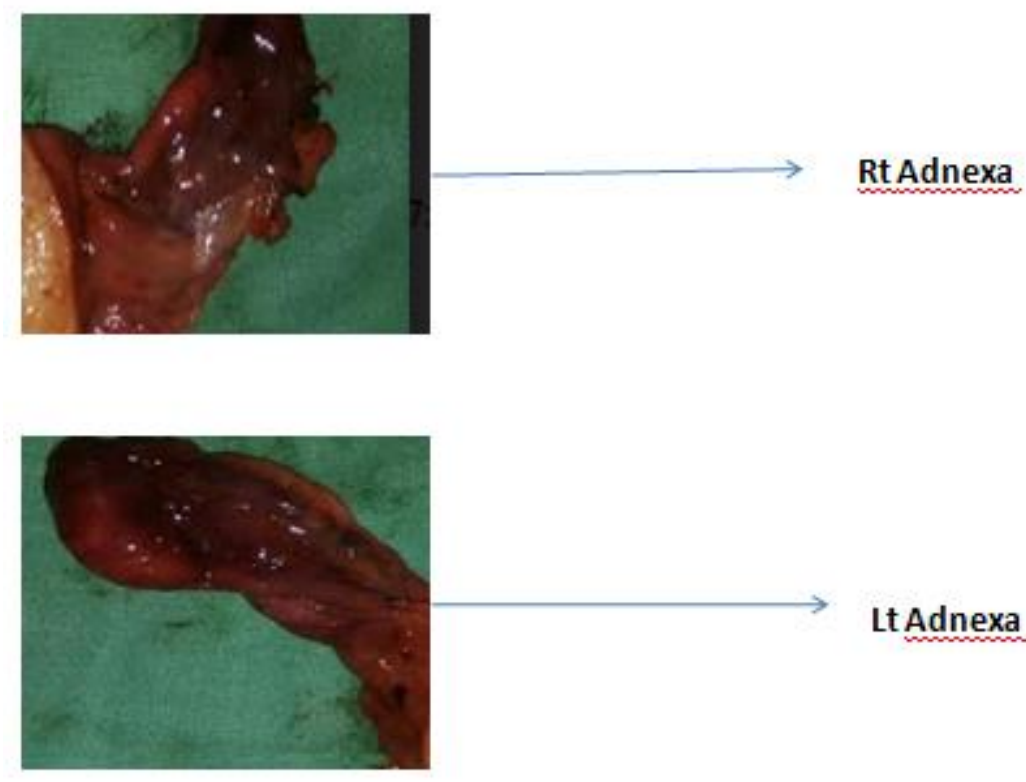

\section{Lt Adnexa}

- $\quad$ section from the growth - highly grade malignant tumor consists of spindloid to oval pleomorphic cells present in small fasicles , arranged in whroled pattern ,separated by fibrous tissue

- $\quad 40-45$ / HPF mitotic figures . Both typical and atypical mitotic figures noted

- $\quad$ necrosis seen $(<50 \%),>50 \%$ myo-invasion, uterine serosa free of tumor

- IMP - high grade malignant spindle cell neoplasm

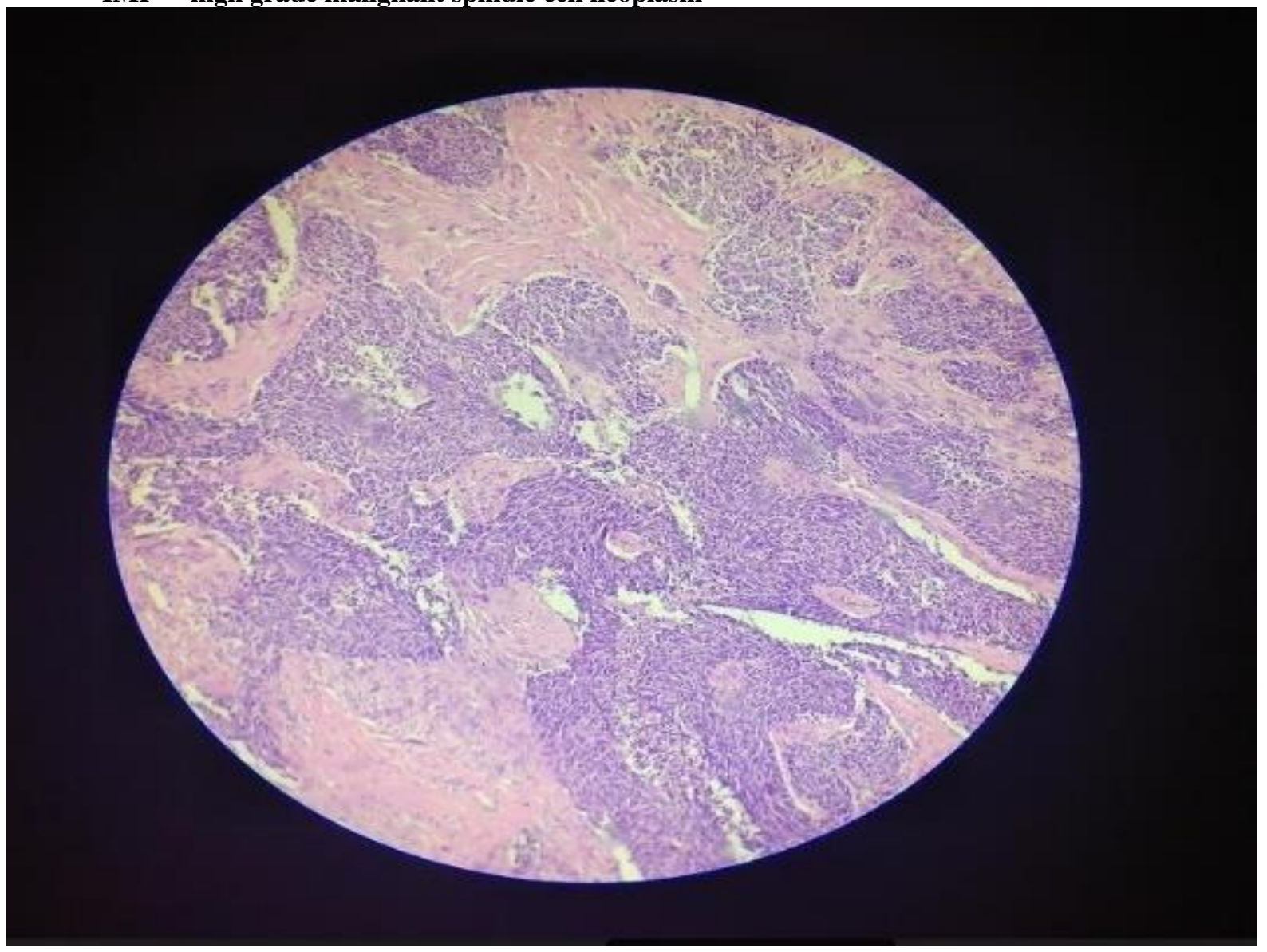




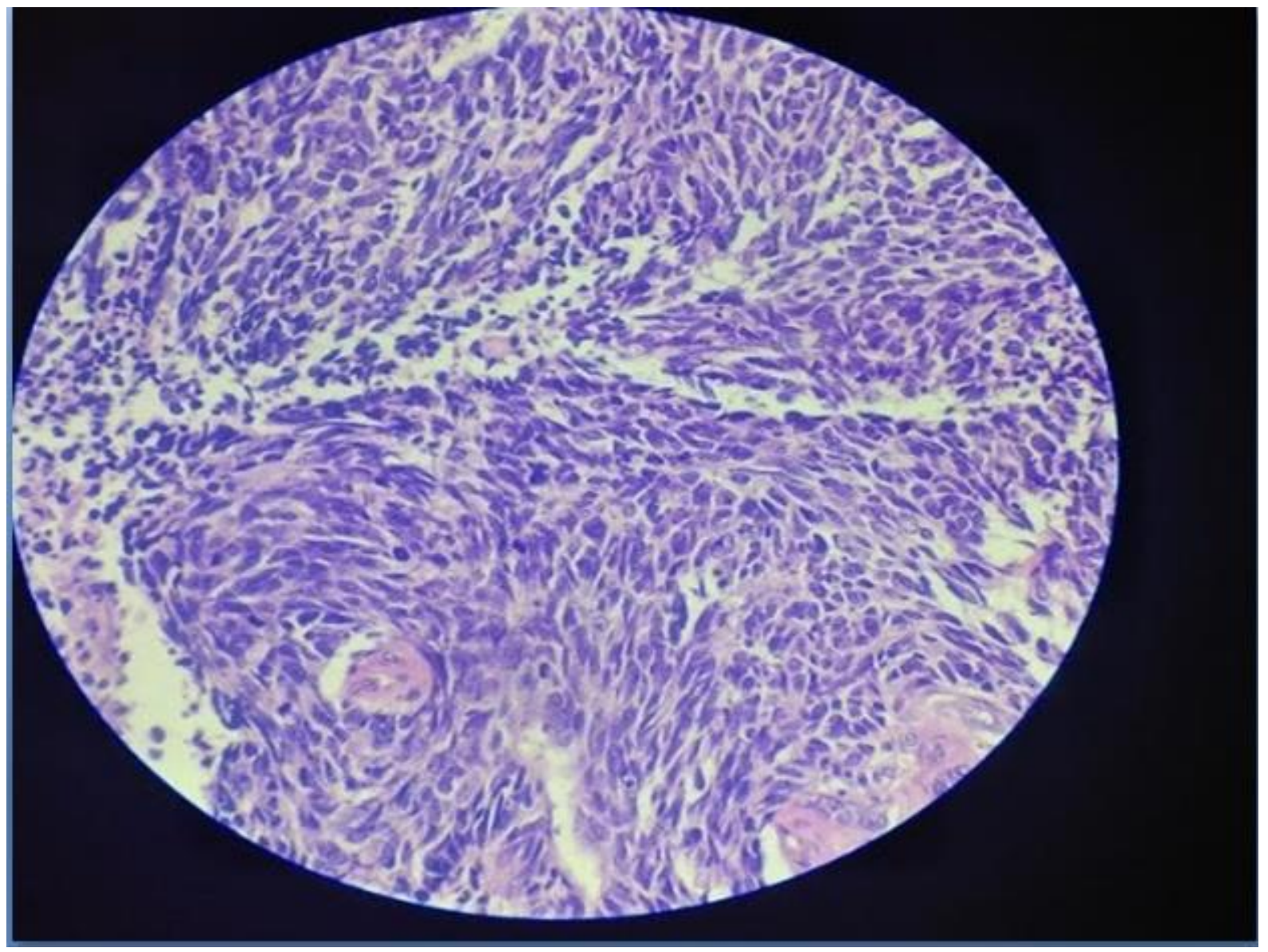

- D/D - poorly differentiated carcinoma leimyosarcoma undifferentiated sarcoma uterine stromal cell sarcoma carcinosarcoma

\section{IHC done}

- $\quad$ SMA, CD10, CK, S1OO

- SMA -NON REACTIVE SCORE 0

- $\quad$ CD-10 - IMMUNO REACTIVE 1+

- S100 - NONREACTIVE

- CK - IMMUNO-REACTIVE3+

- WT-1 - NON REACTIVE

- PAX-8- NON- REACTIVE

- IMP- poorly differentiated carcinoma with spindle cell morphology, native endometrial tissue Post op usg - normal usg of adomen and pelvis

Planning CT and chest xray - normal

DIAGNOSIS - HIGH GRADE POORLY DIFFERENTIATED ENDOMETRIAL CARCINOMA MORPHOLOGY STAGE IB (sarcomatoid variant of endometrial carcinoma)

Treat ment-planned for adjuvant RT(3drt) with CT

\section{CASE -2} presently receiving EBRT

A 63 yrs female presented to opd c/o-pmb- 10 days o/h p6lcb 40yrs, menopause 20yrs

G/E-NAD

P/D- NAD

P/S- cervix flushed with endocervicitis

$\mathrm{P} / \mathrm{V}$ - ut rv, para free, $\mathrm{b} / \mathrm{l}$ fornices free 
Investigations- USG- bulky uterus $(8.6 \times 4.8 \times 6.1 \mathrm{~cm})$ with hyperplastic endometrium

$$
\text { ET- } 30 \mathrm{~mm}
$$

Ovaries-not imaged,No adnexal; mass

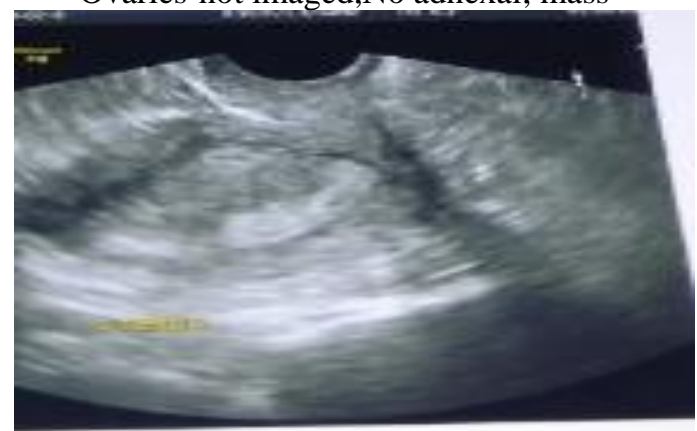

MR I - findings reveals a enhancing mass lesion in the anterior fundic region \& involving anterior myometrium( $>50 \%)$ with peri uterine fat plane and sparing b/l parametrium,sparing cervix, no enlarged lymphadenopathy, normal MRIevaluation of the upper abdomen, finding consistent with ca endometrium.

EB- invasive squamous cell carcinoma cevix,G2

PLAN - COMPREHENSIVE SURGICAL STAGING

IOP-no free fluid

Uterus 8 wks size, b/l adnexa normal

All lymph nodes enlarged

All abdominal organs normal

Omentum normal, mild adhesion

c/s- $5 \times 6 \mathrm{~cm}$ mass in the fundus, with $>50 \%$ myoinvasion, endo cervix not involved

PROCEDURE - TYPE II RADICAL HYSTERECTOMY+BSO+BPLND

adhesiolysis

perItoneal washings taken

adequate parametrium, vaginal margin taken, hysterctomy done

HISTOPATHOLOGY - gross - cut open section uterus- growth present in fundus $3 \times 2 \times 1$, no myometrial invasion, myometrial thickness free of tumor. Lymphnode isolated lar rgest $1.2 \mathrm{~cm}$ diemeter.

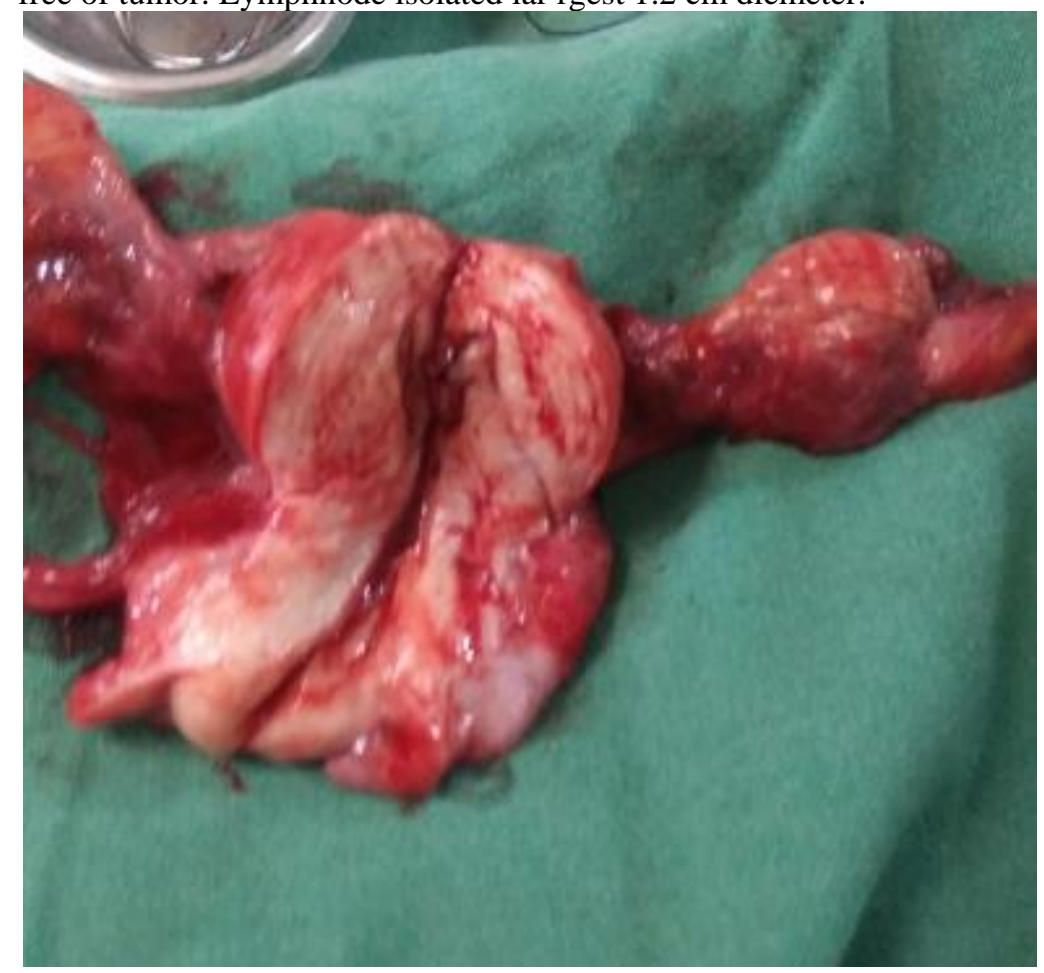

High grade, depth of inavasion. ,50\%, lvsi-ve,b/l para and b/l adnexa free of tumor, cx- chronic cervicitis

Imp-features suggestive of undifferentiated high grade sarcoma

d/d- epitheliod leiomyosarcoma 
poorly differentiated carcinoma

Ihc - panel CK3+VE, EMA - VE,CD10-VE, SMA-VE

DESMIN-VE, Ki-67 -VE3+

CD-117- VE, INHIBIN- VE, CALRETININ-VE,SOX--VE
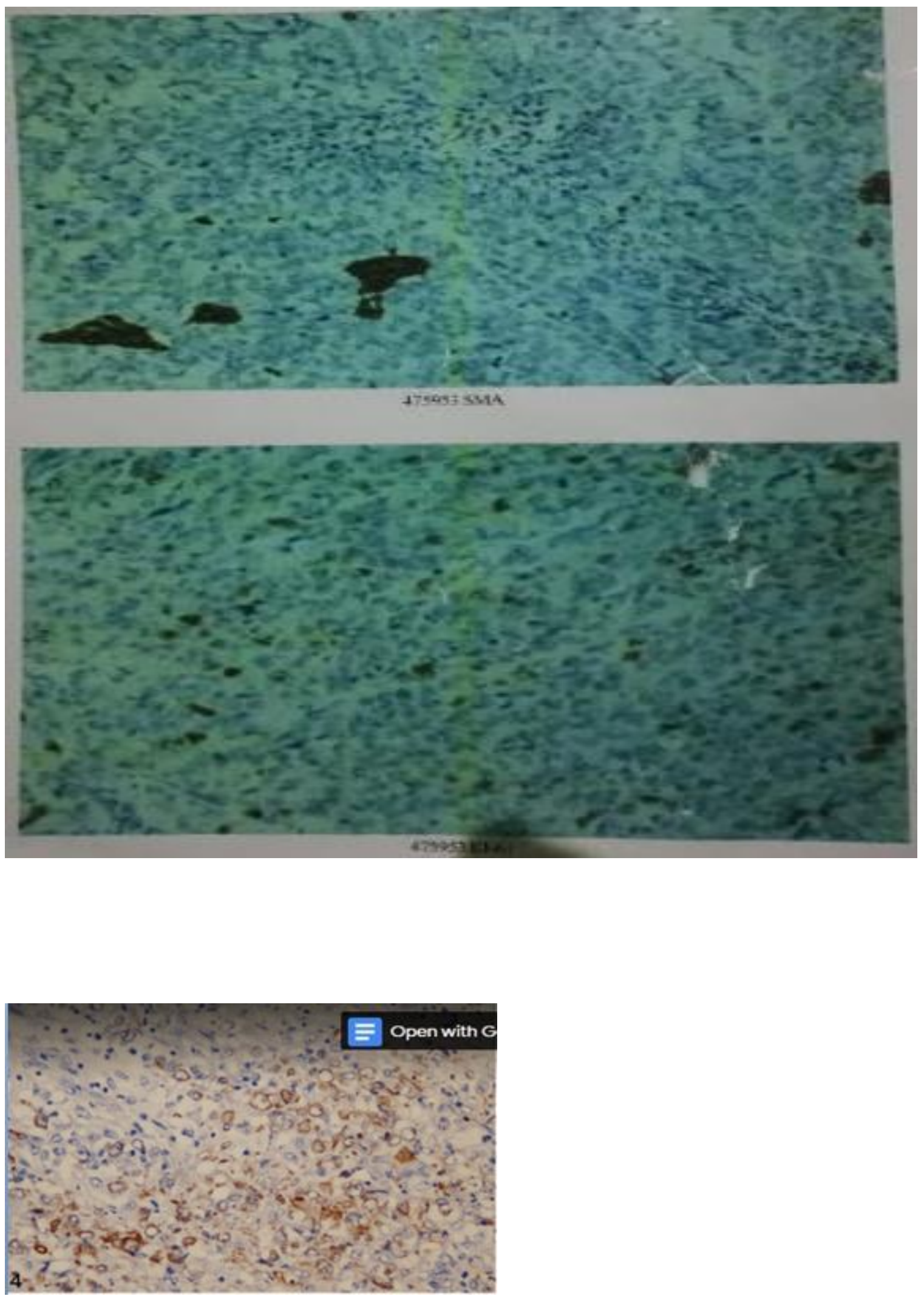

Focal and intense positivity of of cytokeratin in poorly differentiated carcinoma 


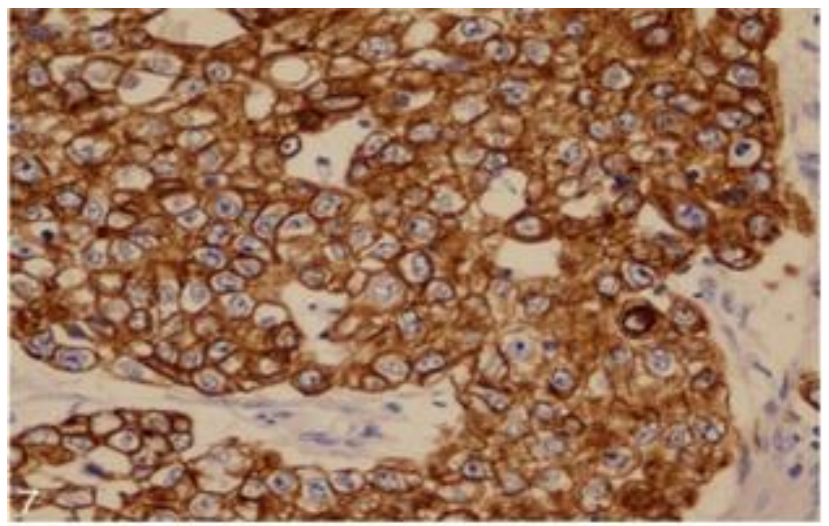

Intense diffuse positivity of cytokeratin in a poorly differentiated carcinoma

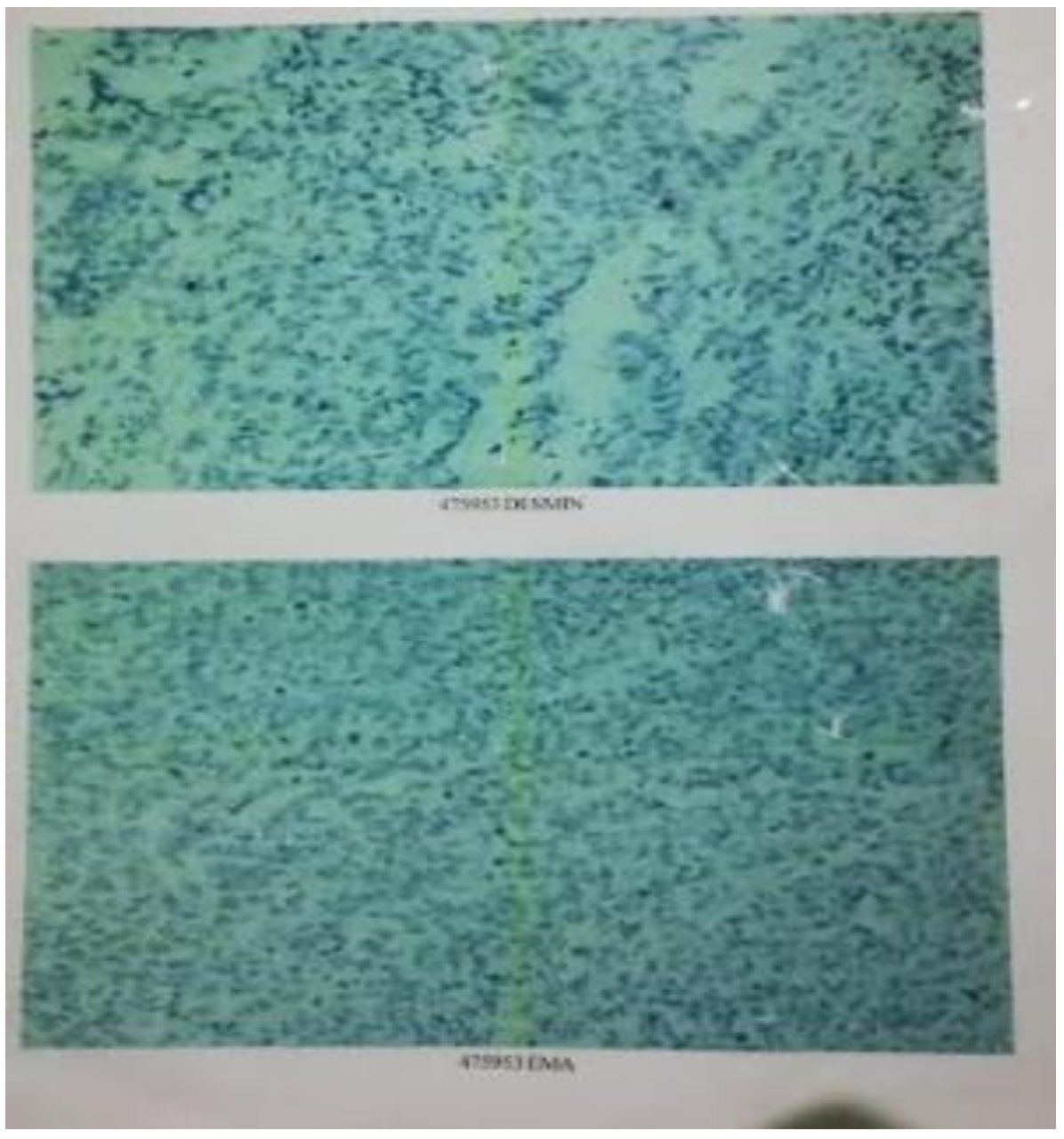




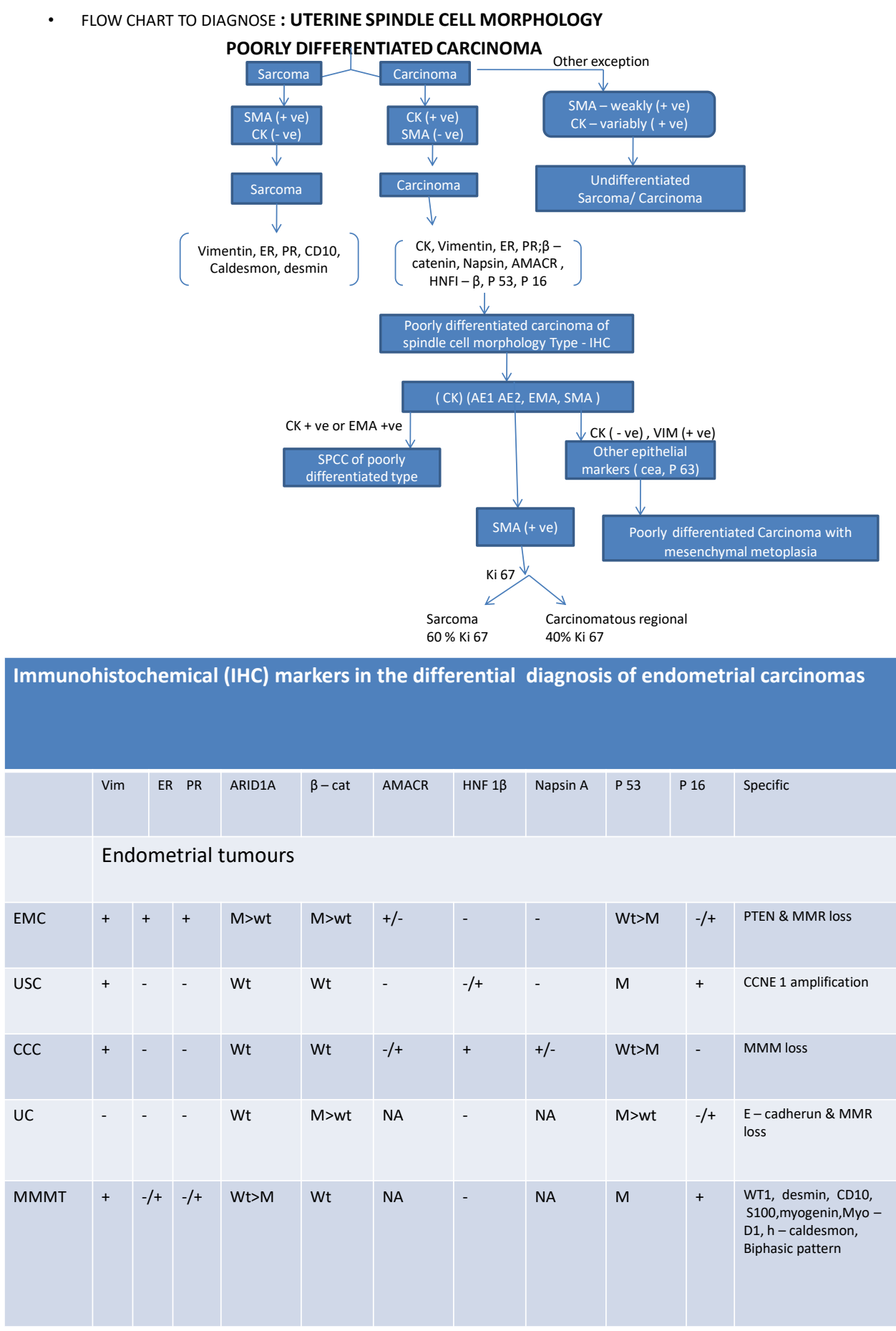

\section{IHC in favour uterine carcinoma}

- CK- CK-(AE-1/AE-3,8,18 )is frequently positive, CK- 18 more frequently positive(1)

- CAM5.2 (2)

- EMA- usually focally positive, very rarely diffusely positive

special emphasis should be give to intensity of staining of keratin AND EMA than the percentage of staining(3)

- $\quad$ ER/PR- CONFLICTING DATA(1)

- Vimentin -ve or may be focally positive,

- Focally positive CD-10.

- Focal positivity for S-100, CD- 56

OTHER IHC MARKERS

- Synaptophysin, chromogranin,CD56- usually -ve, may be focally positive $(4,5)$

- P-16-is diffusely / stronglypositive +/_ve 
- $\quad$ P53 wt- weakly nuclear positivity seen

SPECIFIC- loss of , E- CADHERIN $(4,5)$

Poorly differentiated carcinoma - is completely negative SMA, desmin ,HMB-45

Differential diagnosis

\section{UNDIFFERENTIATED STROMAL SARCOMA /PDEC(9)}

CK- Negative, negative of epithelial markers

strongly positive for CD-10,
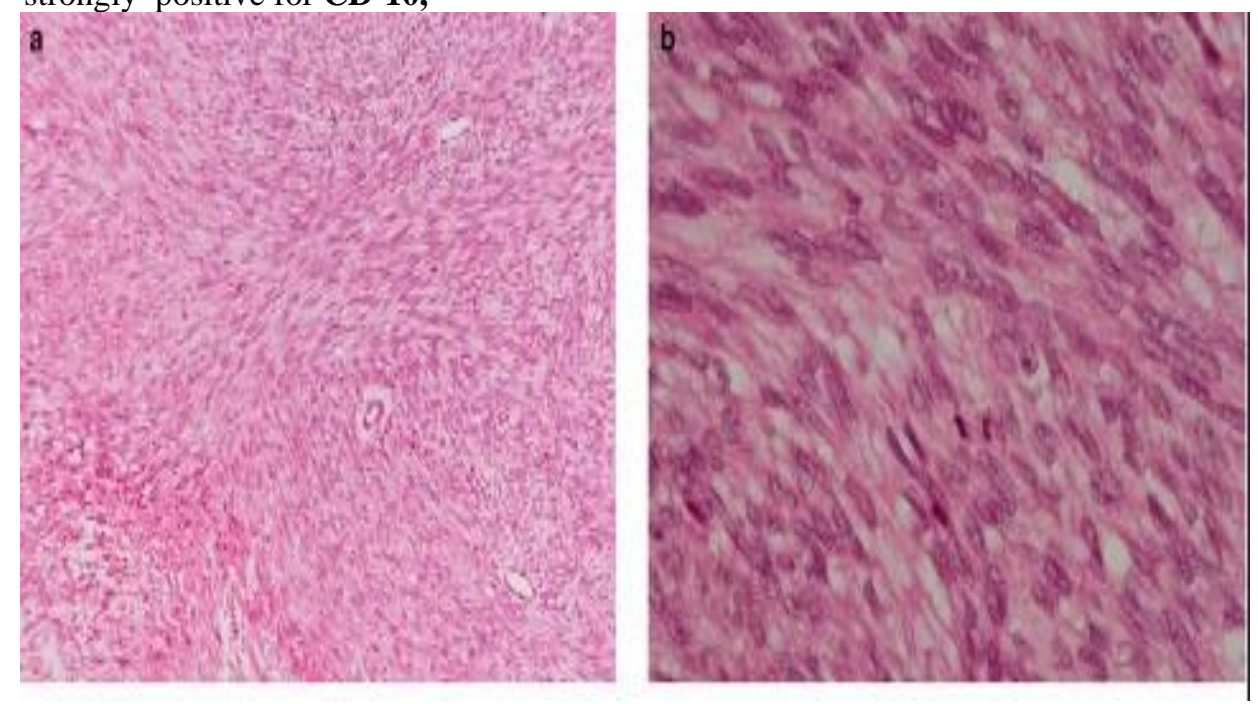

\section{LEIOMYOSARCOMA /UEC(2)}

- $\mathrm{CD} 10$ - -VE

- Sma-+ve

- Desmin+ve

- Caldesmon +ve

- Negative - Cytokeratin except the epetheloid leimyosarcoma may be focally postive

- RHABDOMYOSARCOMA / UEC (SPINDLE CELL MORHOLOGY)(10)

- Uniform spindle cell with a herring bone pattern

- Negative or else focal reactive FOR CK

- SMA -+VE

- DESMIN+VE, MYOGLOBIN+, CALDESMON

- +ve in spccBAF-47(INI-1) this protein is lost in rhabdoid tumor,

Carcinosarcoma / PDEC(SPINDLE CELL MORPHOLOGY)(8)

there is disinct compartmentalisation of the carcinoma and sarcomatous component.There are presence of heterologous elements ihc +VE WT-1/P53 is helpful indifferentiating from spindle carcinoma.Morphological features is enough for diagnosis

uec/ grade 3 endometrial (7)

Morphology UEC

Growth Diffuse

Glands absent

tumor area

\section{Cords trabeculae}

Component

Cohesive growth

$\begin{array}{cc}\text { Rhabdoid } & \text { may present } \\ \text { Myxoid } & \text { may be present } \\ \text { IHC - PANCYTOKERATIN patchy /focal } \\ \text { EMA } & \text { patchy/focal } \\ \text { ER/PR } & \text { focal }\end{array}$

\author{
ENDOMETROID \\ solid and glandular \\ present in $1-4 \%$ of
}

intermingled demarcation cohesive

absent

absent

diffuse(b)

diffuse(b)

diffuse(b)

Review literature - 
- Poorly differentiated carcinoma is a type II endometrial cancer, about $20 \%$ of endometrial cancer(1)

\section{Types of Endometrial Cancer (EC)}
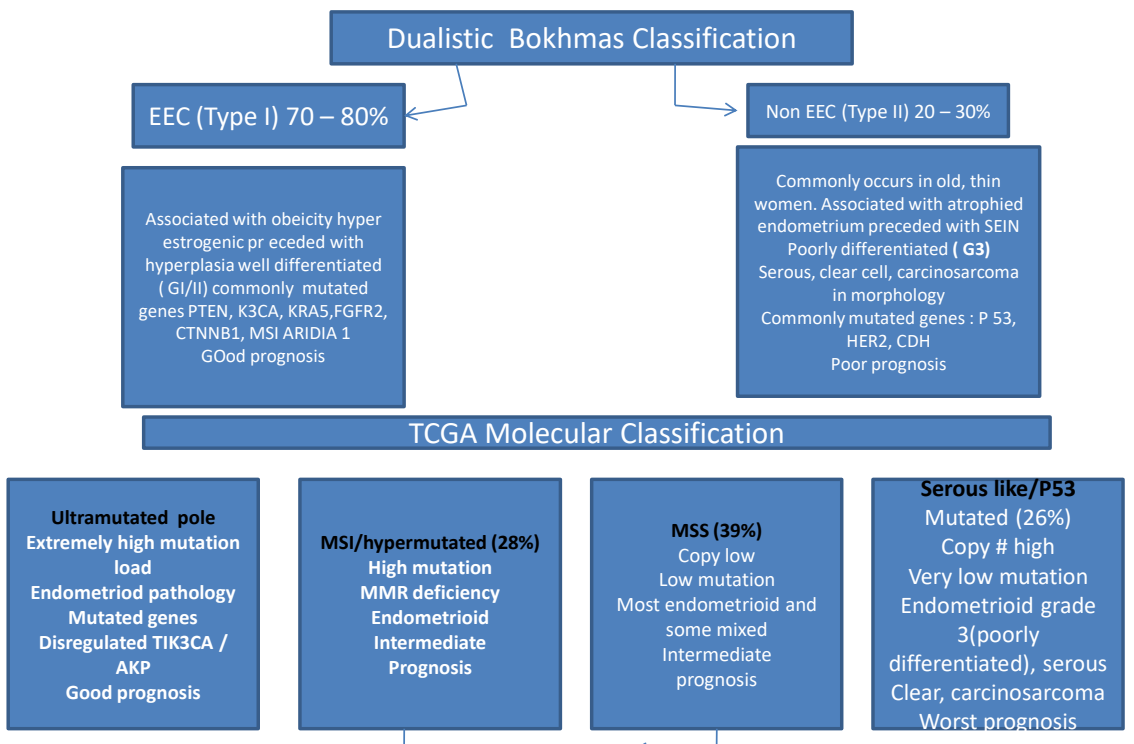

FGRSGFR2

- This group is also associated with hereditary non-polyposis colorectal carcinoma(lynch syndrome).Significant number of patients display loss of 1 more DNA mismatch repair.

\section{Pathogenesis}

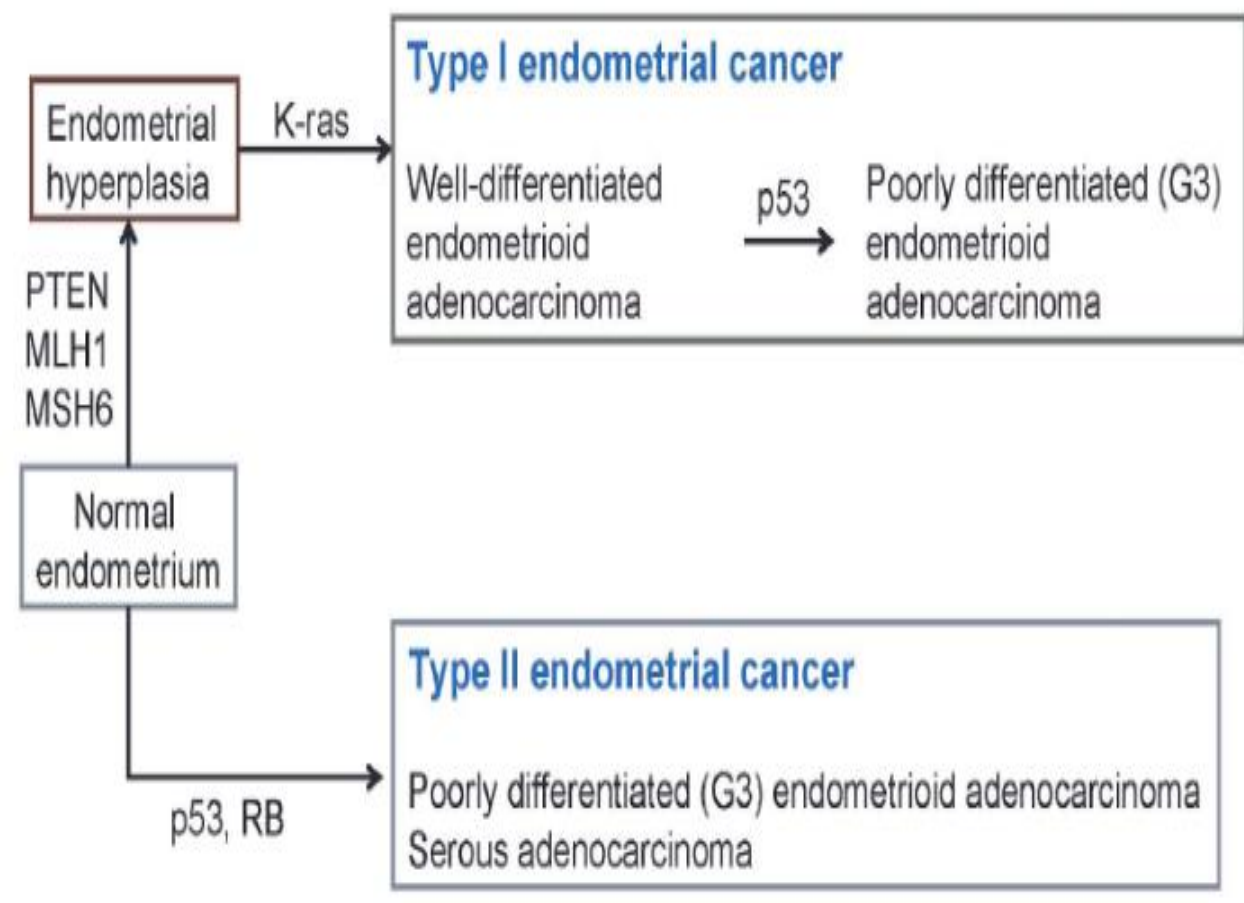




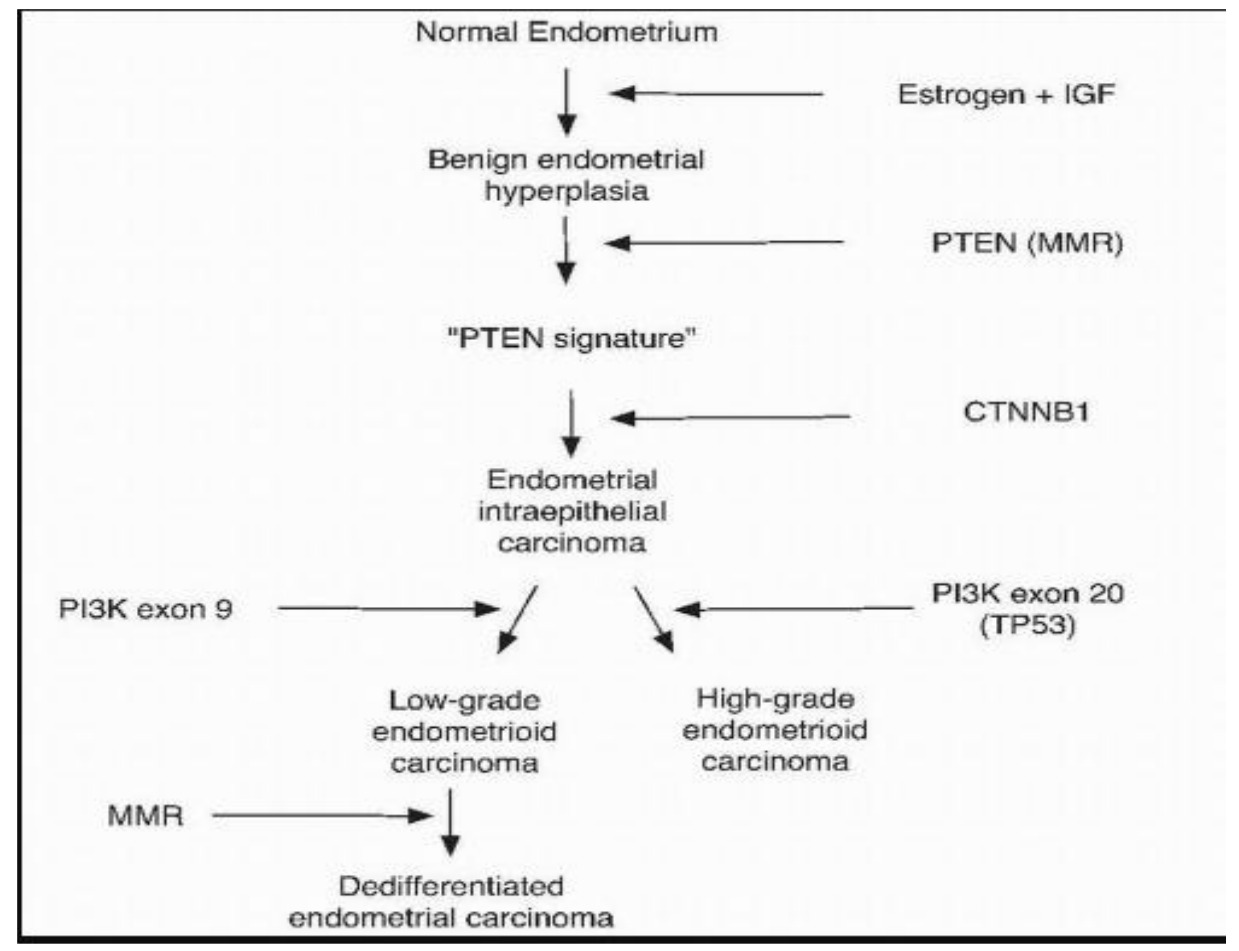

About half present in higher stage(III AND IV)(2)

GROSS PATHOLOGY - may present as polypoidal fleshy masses with evident of necrosis

Usually involves the lower uterine segment

MICROSCOPICALLY - solid pattern less growth of medium size relatively monomorphic round or polygonal epithelial cells that lack glandular differentiation. Sometimes pleomorphism detected(5)

Very occasionally- spindle cell pattern, alveolar pattern is found in uc $(3,4,5)$

\section{PROGNOSIS}

- Prognosis is poor irrespective of age, stage, presence an number of tumor infiltrating lymphocytes, any rhabdoid or spindloid morphology

- Prognosis depends in MMR mutation,up AND DOWNREGULATION OF MI -RNAS due abberant methylation thus making it sensitive to taxanes.(6)

- $\quad$ P53 mutant type i.e mss high copy nos, worse pognosis

- variability in expression or of cell adhesive glycoprotein loss e- cadherin can determine the prognosis

- Other HER-2, ECAM poor prognosis

- $\quad$ ploidy status of these tumors usually aneuploid

- High grade g3 has high chances of recurrences in comparison to grade1 and 2.

- However there are instances where grade3 endometrial cancers do show a good prognosis

- The fact is validated by a japanese study taking 12 prognostic factor of grade 3 carcinoma into consideration . Among them absence of adnexal metastasis, no cervical involvement, low pre-surgery CA602 and a low CA-19.9 carried a favourable prognosis .(7)

\section{MANAGEMENT -}

Currently there is no difference in management of grade 3 endometroid, undifferentiated and dedifferentiated endometrial carcinoma all are HIGH RISK CATEGORY (8)

- $\quad$ TAH +BSO and chemotherapy and radiotherapy

\section{OR}

- $\quad$ surgical staging(TAH+BSO+ PARTIAL OMENTECTOMY+PERITONEAL WASHINGS+ B/LPLND+B/LPAND FOLLOWED BY EBRT +CT)

- PORTEC-3 TRIAL on HIGH RISK PATIENTS IN STAGE I-II OR STAGE III, revealed higher failure free survival and decrease in pelvic recurrences a of chemoradiotherapy( two concurrent cycles of cisplatin in weeks 1 and 4 of ebrt , followed by four cycles of carboplatin and paclitaxel) than EBRT alone 
- GOG 258 AND GOG 249 greater evidence support EBRT+ CHEMOTHERAPY

- E-cadherin is an independent predictor of survival of endometrial cancer, regardless of histology. $(9,11)$

- Loss ofE- cadherin ,is of poor prognosis, advance stage, poor differentiation.

- E- caderin has a central role in organisation of epithelial structure(cohesiveness) it also regulates apoptosis.

- $\quad$ Presence of e- cadherin almost precludes invasiveness .

- It is also related tumor budding(11)

Miyamato etal studied and compared the expression of E- CADHERIN and cytoplasmic E-cadherin related molecules ( alphacatenin, beta catenin, gamma catenin, in well differentiated and poorly differentiated carcinoma .(11)

- They reported significant difference in expression between the above two histological types.

- The recently used drug DASATINIB Increase the expression of e-cadherin , by repressing slug-mrna

- The literatures reveals the presence of E-cadherin

Increases the sensitivity to EGFR inhibitors (10)

Immunotherapy in poorly differentiated endometrial cancer

- Recent clinical phase II Trials on Nivolumab and Iplimumab in undifferentiated endometrial cancer

- The addition of pembrolizumab with paclitaxel and carboplatin is undifferentiated carcinoma in phase IIItrial

Immune checkmpoint inhibitors (ANTIPD-1/PD-1 antibody,ANTI CTL-4antibody) could be effective in treatment of poorly differentiated endometrial carcinoma and the presence of MSI may be a bio-marker for good response to PD-L1 immunotherapy

FOLLOW UP -

- these patients should be on close follow up

- 3 monthly for first- 3 months with usg, ct vault examination and cytology

- 6 monthly for next 3 yrys

- thereafter once a year

CONCLUSION - After corelating the hps and ihc we coulfd reach the final diagnosis of poorly differentiated carcinoma of endometrium , accordingly planned her treatment

PURPOSE - The case series will throw some light in area of diagnosis and management of poorly differentiated carcinoma endometrium, keeping in view of its variety of hps morphology a broad differential diagnosis. To help in diagnosis of a poorly differentiated carcinoma, particularly the sarcomatoid or the spindloid morphology can be sometimes be confuse with sarcoma i.e undifferentiated sarcoma or a leimyosarcomaor a rhabdomyosarcoma.Due to the confusing histopathology picture, IHC staining and interpretation may be required, to reach the final diagnosis.

\section{REFERENCES}

[1] Narita, sato a ettal undifferentiated carcinoma IHC EUR JOURNA

[2] Tafe lg, garg etal mod pathology

[3] Abeler etal clinical study of 31 caseson indifferentiated endometrial cancer

[4] Pathology outlines nalini gupta, bmJSpindle cell carcinoma uterus - HIROSE ETAL 1987

[5] Fifta sf etal spcc a cytopathological review)

[6] Lewis js, spindle cell lesions)

[7] Altrubisiietal amj pathology 2016

[8] Bartusch ac, manuel adv ant pathology 2015

[9] Olive e young etal amj pathology

[10] Garag k,soslow RA, lynch syndrome and endometrial carcinoma j. clinical pathology)

[11] Meral koyyouncho etal ,journal of gynaecology oncolgy)

[12] Tips am plaxe sc, endometroid carcinoma with low grade spindle component

\section{AUTHORS}

First Author - S.Spattnaik,J.J Mohapatra, J.Parija, L.Sarangi,R.Das, N.Rout,S.K Giri,S.Samantray, N.Panda,P.Devi, P.K Das B.L Nayak, M.Rmohapatra,A.K Padhi,S.Panda,

Dept - Gynaecology Oncology, Institute- Ahrcc, City Cuttack, State -Odisha, Country India 
